voltage and current. The power consumed in a resistance is stated as an axiom, since the real nature and dimensions of voltage and eurrent are not revealed until much later. The book is indeed more valuable for the technologist wishing to tie up loose ends of his electrical knowledge than it would be for the young student aiming to grasp the fundamentals of his subject. For him, Coulomb's law of forces between electric charges remains the natural starting point of his subject. The best method of introducing the concept of the magnetic field is perhaps more controversial; but it is doubtful if many teachers of electrical engineering will be prepared to relegate mechanical forces to the secondary position which they occupy in this book.

About half the book is devoted to an exposition of selected problems. The author's self-denying ordinance with regard to the use of mathematics prevents the study of these problems to any great depth, but the reader's interest is maintained throughout.

Electrical engineers in Britain should be grateful to Dr. Jolley for the most attractive translation of this work and to the Cleaver-Hume Press for the publication of it. No misprints were found, the contrast of print and paper are excellent and the diagrams clear.

A. H. M. ARNold

\section{AN ATLAS FOR SPECTROCHEMICAL ANALYSIS}

\section{Atlas of Analysis Lines}

Prepared by Halina Jaffe, with the technical assistance of N. Turkeltaub. Pp. $13+50$ plates. (London: Hilger and Watts, Ltd., 1962.) £26 58 .

7 HE publication by Halina Jaffe presents the enlarged iron spectrum in three ranges, $2478 \AA$.$3499 \AA ., 3324 \AA .-8943 \AA$, and $3981 \AA$..-5350 $\AA$., the last range being taken with glass optics. The spectra are presented on 50 cards, the iron are spectrum being in the centre, the persistent lines of 69 elements having been added manually on either side of it.

In assessing the value of this Atlas, comparison will naturally be made with its well-known predecessor by Gatterer and Junkes, and also with others that have appeared from time to time in text-books on the subject, of which that by Brode (Chemical Spectroscopy) is probably the most widely used. Jaffe's Atlas has advantages over either of these but at the same time is not devoid of its own limitations.

The charts are of handy size and are particularly suitable for use with Hilger instruments. 'The author emphasizes this, and specifies type and number of spectrograph and projector used in the production of the charts; using other projectors by the same maker, however, they are somewhat less convenient. The arc lines appear as black lines on a white ground as in a normal spectrogram, at a degree of enlargement well chosen for the purpose. Two tables are included which list the most persistent lines of the 69 elements which have been included in the charts, in order of element and in order of wave-length, respectively. These are not intended to be comprehensive wavelength tables; in fact only two lines are included for copper and silver, for example, up to eleven for dysprosium. A wave-length scale is lacking, which seems a rather serious omission; if one could have been included in Ångstrom units for the shorter wave-length range and in five Angstrom units for the higher ranges, it would have been a considerable advantage. The inclusion of a range using glass optics is to be commended for those who have instruments with this additional equipment; hitherto workers in this medium have been rather ignored when aids of this type have been offered.

On general grounds, the spectra suffer from the inherent 'graininess' of the photographic emulsion, a defect that could scarcely be entirely avoided. The wisdom of glossy photographs on thin card for use in a laboratory is questionable; though users can over. come the flimsiness by mounting each card on a thicker backing, a matt finish would probably have been more serviceable.

The price of $£ 26$ may seem high, but this is probably justified when one considers the man-hours necessary to make a similar series of charts, even if it be confined to the range more commonly used in any particular laboratory. The ready comparison of the charts in this Atlas with spectrograms prepared with Hilger equipment should make it worthy of a place in the spectrographic laboratories where such equipment is to be found, and by no means out of place where other equipment is in use.

\section{J. A. C. MoCugllaind}

\section{$\pi$-ELECTRON CHEMISTRY}

Molecular Orbital Theory for Organic Chemists

By Prof. Andrew Streitwieser, Jr. Pp. xvi +489 . (New York and London: John Wiley and Sons, Inc., 1961.) 1098 .

7 HIS is a book for which there has long been a need. For although the greatest successes of the theoretical chermists since the advent of the wave equation in 1926 have been in the field of organic chemistry, there has hitherto been no simple account of the whole topic.

Prof. Streitwieser is well qualified to write such an account; for he combines both the experience of the experimental chemist, who knows how hard it is to sort out a genuine measure of the charge around each atom of a molecule, and also the ability of the theoretical chemist, since he has made some notable contributions to our store of calculated quantities. It is this balance between theory and experiment which must count as one of the strongest points in favour of this book. The literature abounds in 'bastard mathematics'-by which I mean poorquality mathematics which serves practically no purpose because it solves no problems. There is none of that here.

The author has concentrated on $\pi$-electron chemistry - which seems for him to be almost synonymous with organic chemistry - and he deals only with the molecular-orbital approximation. This is wise, because by far the greatest successes and the widest applications are within this approximation. $\mathrm{He}$ also concentrates almost entirely on the simplest Hückel theory, with no overlap integrals and no allowance for electron exchange (except in the development of the $\omega$-technique, largely developed by the author himself). As a result, the account is excellent for ground states, but becomes much loss convincing in those few places where excited states are involved. But it is quite amazing to see what can be done, even with so gross an approximation. 